\title{
Finite element analysis of hypervelocity impact behaviour of CFRP-Al/HC sandwich panel
}

\author{
Vaibhav A. Phadnis ${ }^{1}$ and Vadim V. Silberschmidt ${ }^{2, a}$ \\ ${ }^{1}$ AMRC with Boeing, The University of Sheffield, UK \\ ${ }^{2}$ Wolfson School of Mechanical and Manufacturing Engineering, Loughborough University, Loughborough, UK
}

\begin{abstract}
The mechanical response of CFRP-Al/HC (carbon fibre-reinforced/epoxy composite face sheets with Al honeycomb core) sandwich panels to hyper-velocity impact (up to $1 \mathrm{~km} / \mathrm{s}$ ) is studied using a finite-element model developed in ABAQUS/Explicit. The intraply damage of CFRP face sheets is analysed by mean of a user-defined material model (VUMAT) employing a combination of Hashin and Puck criteria, delamination modelled using cohesive-zone elements. The damaged $\mathrm{Al} / \mathrm{HC}$ core is assessed on the basis of a Johnson Cook dynamic failure model while its hydrodynamic response is captured using the Mie-Gruneisen equation of state. The results obtained with the developed finite-element model showed a reasonable correlation to experimental damage patterns. The surface peeling of both face sheets was evident, with a significant delamination around the impact location accompanied by crushing $\mathrm{HC}$ core.
\end{abstract}

\section{Introduction}

Advanced composite materials such as CFRP/Al-HC (carbon fibre-reinforced plastic face-sheets/aluminium honeycomb core) sandwich panels typically find their application in satellite structures due to their excellent mechanical properties such as high specific strength, stiffness and ability to provide tailored properties. In the absence of proper shielding, these structures can be often exposed to impacts by space debris. Many satellites already employ honeycomb sandwich panels with CFRP face-sheets covered by multi-layer thermal insulation as external walls [1]. Furthermore, FRPs are often used in the construction of spacecraft components such as antenna struts, low distortion frames and lightweight robotic arms [2-5].

Still, many concerns remain about the possibility of employing polymer-matrix composites as the primary satellite structure for long-duration missions because of severe environmental conditions encountered in space. One of the major problems is related to the vulnerability to impact damage from meteoroids and man-made space debris that populate the near-Earth orbital environment. Such particles travel at speeds of several $\mathrm{km} / \mathrm{s}$, thus presenting a serious hazard for existing satellites. Previous researchers found that damage due to high velocity impacts (HVIs) on composite materials consists of an impact crater, surface peeling of the outer laminae on both the front and rear side of the plate and a significant internal delamination around the impact point, that can lead to severe degradation of the material's mechanical strength. Moreover, if a hypervelocity particle has enough energy to fully perforate the impacted plate, a debris cloud emerging from the rear face of the target can significantly

\footnotetext{
${ }^{a}$ Corresponding author: v.silberschmidt@lboro.ac.uk
}

damage adjacent structures and internal components of the spacecraft.

This study focuses on the development of a finite element (FE) model to simulate a HVI behaviour (with velocities up to $1 \mathrm{~km} / \mathrm{s}$ ) of a CFRP-Al/HC sandwich panel using general-purpose FE software Abaqus/Explicit [6]. A user-defined material model (VUMAT) developed to study damage of CFRP face sheets employing combined Hashin and Puck criteria was used, while the interply damage was modelled by means of cohesive-zone elements. A hydrodynamic response of $\mathrm{Al} / \mathrm{HC}$ was captured using a Mie-Gruneisen equation of state and its damage was modelled using a Johnson Cook dynamic failure model. The developed FE model was validated with experimental data from Wicklein et al. [7] and showed a reasonable correlation between damage patterns experimentally and numerically.

\section{Finite-element model}

A quarter of the full geometry of the sandwich plate was modelled owing to its symmetry about relevant planes; this resulted in significantly reduced computational efforts. A steel projectile of $3 \mathrm{~mm}$ diameter was impacted on the sandwich structure with an impact velocity ranging from $700 \mathrm{~m} / \mathrm{s}$ to $1 \mathrm{~km} / \mathrm{s}$. The general contact algorithm in ABAQUS/explicit[6] was used to simulate contact conditions between the projectile and the composite facesheets and $\mathrm{Al} / \mathrm{HC}$, and between the layers of CFRP laminate by defining appropriate contact-pair properties. The setup of FE model is shown in Fig. 1.

The elastic response of CFRP laminate was accounted for; it is discussed in Sect. 2.1. An element-deletion approach was used to model a material-erosion process based on initiation and evolution of damage in the meshed elements. Criteria for damage initiation and evolution are discussed in Sect. 2.2. 


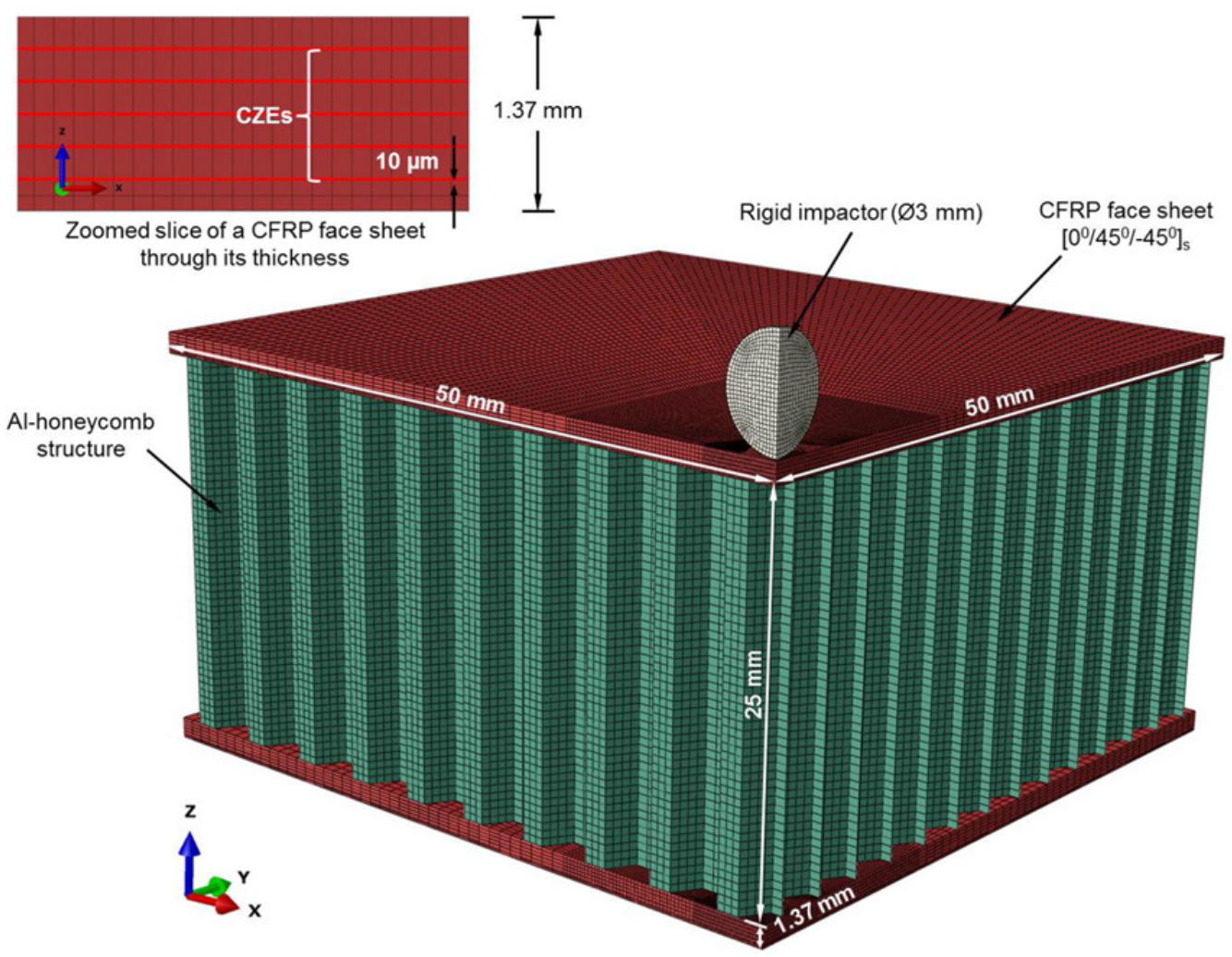

Figure 1. Setup of FE model.

\subsection{Response of UD composite laminate}

An elastic stress-strain relationship for a UD composite ply was introduced assuming the orthotropic damaged elasticity response. Its constitutive behaviour before damage is expressed using a generalised Hooke's law in the tensorial form:

$$
\{\sigma\}=\mathbf{C}\{\epsilon\} .
$$

The effect of strain-rate on elastic moduli and strength was defined in order to accurately account for a high strain-rate response of the laminated composite material. It should be noted that for carbon/epoxy-like systems, where carbon fibres are brittle and show no strain-rate dependence, the strain-hardening at higher loading rates is entirely due to epoxy matrix; while in glass/epoxy like systems both glass fibres and epoxy matrix may contribute towards this.

The strain-rate effects in the elastic and shear moduli are defined by the following expressions:

$$
\begin{aligned}
& E(\dot{\epsilon})=E\left(\dot{\epsilon}_{0}\right)+\left[m_{e} \cdot \log \left(\frac{\dot{\epsilon}}{\dot{\epsilon}_{0}}\right)+1\right] \\
& G(\dot{\gamma})=G\left(\dot{\gamma}_{0}\right)+\left[m_{s} \cdot \log \left(\frac{\dot{\gamma}}{\dot{\gamma}_{0}}\right)+1\right],
\end{aligned}
$$

where $E(\dot{\epsilon})$ and $G(\dot{\gamma})$ are elastic and shear moduli for a given strain rate, $E\left(\dot{\epsilon}_{0}\right)$ and $G\left(\dot{\gamma}_{0}\right)$ are elastic and shear moduli at the reference strain rate $\dot{\epsilon}_{0}$ and $\dot{\gamma}_{0}$, respectively, in three directions and three planes of a $3 \mathrm{D}$ co-ordinate system. $m_{e}$ and $m_{s}$ are the material parameters obtained by fitting a curve to the experimentally obtained data. The effects of strain rate on ply strength are discussed in the following sections where initiation of different damage modes is considered.

\subsubsection{Damage initiation}

Modelling of composites and their damage at a laminate level typically requires input of several parameters, including homogenised ply properties, interply strength and information about the laminate lay-up. Here, a layerby-layer modelling strategy was adopted to capture failure in each ply. This offers several advantages. Firstly, full 3D stress states can be analysed. Typically, FE models of composite deformation involve the use of 2D shell elements to simulate composite plies, which do not allow for accurate representation of stress through the composite thickness.

Secondly, intraply and interply damage can be introduced discretely along with phenomenological models that account for complex interaction between them. Here, a material model offering a combination of Hashin's [7] and Puck's failure criteria [8] was used to implement the advantages of both. The Hashin's criteria were employed to estimate damage in carbon fibres while damage in epoxy matrix was modelled using the Puck's criteria. The empirical forms of these criteria are discussed below.

\section{(a) Fibre damage}

- in tension.

Here, tensile failure of fibres is defined as a function of tensile strength of lamina along the fibre direction as well 
as shear strength in transverse directions (in and out-of plane). The empirical relation is expressed in the following form:

$$
\left(\frac{\sigma_{11}}{X_{1 t}}\right)^{2}+\left(\frac{\sigma_{12}}{S_{12}}\right)^{2}+\left(\frac{\sigma_{13}}{S_{13}}\right)^{2} \geq 1, \quad d_{f t}=1
$$

where $X_{1 t}$ is the tensile strength of a composite in fibre direction, while $S_{12}$ and $S_{13}$ are its shear strengths in 1-2 and 1-3 planes, respectively. $d_{f t}$ is a scalar damage variable indicating fibre damage in tension such that $0 \leq d_{f t} \leq 1,0$ represents no damage, while 1 indicates fully-damaged state; this nomenclature applies to all damage modes discussed below. Here, $X_{1_{\digamma}}$ is assumed equal to its quasi-static magnitude since fibres show negligible or no strain-rate dependence at high loading rates.

- in compression.

Longitudinal compressive failure of composites is driven by shear mechanisms in an intricate way. A first crack may be started by shear fracture of fibres followed by rotation and in-plane shearing of the matrix at the crack tip, which in turn promotes kink-band development. The empirical relation for this damage mode is expressed in the following form

$$
\left(\frac{\sigma_{11}}{K(\dot{\epsilon}) X_{1 c}^{q s}}\right)+\left(\frac{\sigma_{12}}{K(\dot{\gamma}) X_{12}^{q s}}\right)+\left(\frac{\sigma_{13}}{K(\dot{\gamma}) X_{13}^{q s}}\right) \geq 1, \quad d_{f c}=1 .
$$

The scaling parameter $K$ is defined as:

$$
K=\frac{S_{12}^{d y n}\left(\dot{\gamma}_{12}\right)}{S_{12}^{q s}}=\frac{S_{13}^{d y n}\left(\dot{\gamma}_{13}\right)}{S_{13}^{q s}} .
$$

Here, $S_{12}^{d y n}(\dot{\gamma})$ and $S_{13}^{d y n}(\dot{\gamma})$ are in-plane and out-of-plane shear strengths of a ply in dynamic loading condition at a given strain rate and $S_{12}^{q s}$ and $S_{23}^{q s}$ are its shear strengths in quasi-static loading condition.

\section{(b) Matrix damage.}

The phenomenological matrix-failure criteria for 3D QS failure analysis of UD composites were previously formulated by Puck and Schürmann [8]. These criteria forms a basis of the criterion presented here. The applicability of the originally proposed tensile and compressive failure criteria for quasi-static loading conditions was extended to the model material's response under dynamic loading.

The polymer matrix material in a CFRP composite demonstrates strain-rate sensitivity at high strain rates $\left(\sim 10^{3} / s\right)$ which are typical for high-velocity impact events. This effect becomes significant, particularly in transverse directions, where the polymer matrix is a primary load bearing. An approach, similar to that employed in modeling compressive failure of fibres was adopted here to account for strain-rate sensitivity. The following criteria were proposed to model failure in the

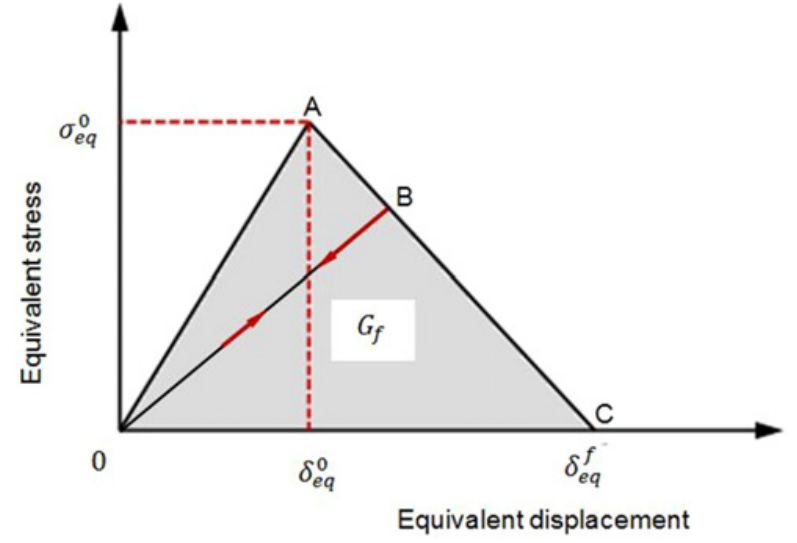

Figure 2. Concept of equivalent stress vs. equivalent displacement in evolution of damage variable.

matrix material in compression and tension:

$$
\begin{aligned}
& \left(\frac{\sigma_{22}}{K(\dot{\epsilon}) X_{2 t}^{q s}}\right)^{2}+\left(\frac{\sigma_{12}}{K(\dot{\gamma}) S_{12}^{q s}}\right)^{2}+\left(\frac{\sigma_{23}}{K(\dot{\gamma}) S_{23}^{q s}}\right)^{2} \geq 1 ; \\
& \sigma_{22}+\sigma_{33}>0, \quad d_{m t}=1 \ldots \text { (tensile failure) }, \\
& \sigma_{22}+\sigma_{33}<0, \quad d_{m c}=1 \ldots \text { (compressive failure). }
\end{aligned}
$$

\subsubsection{Damage evolution}

To alleviate mesh dependency during strain softening, a characteristic length $\left(L_{c}\right)$ of a finite element was introduced into the formulation so that the constitutive law was expressed as a stress-displacement relation as shown in Fig. 2. The positive slope of the stress-displacement curve prior to damage initiation corresponds to a linearelastic material behaviour; while the negative slope after damage initiation is achieved by evolution of damage parameter $d$ as following:

$$
d_{i}=\frac{\epsilon_{e q}^{f i}\left(\epsilon_{e q}-\epsilon_{e q}^{0}\right)}{\epsilon_{e q}\left(\epsilon_{e q}^{f i}-\epsilon_{e q}^{0}\right)} .
$$

where $\epsilon_{e q}^{0}$ is the strain at damage initiation, and $\epsilon_{e q}^{f}$ is the strain at which the material is completely damaged. $i=f_{t}, f_{c}, m_{t}, m_{c}$ corresponds to damage variables associated with failure modes of fibre and matrix material in tension ( $\mathrm{t}$ ) and compression (c). The strain at failure was expressed as,

$$
\epsilon_{e q}^{f}=\frac{2 G_{f}}{\sigma_{e q}^{f} \cdot L_{c}},
$$

where $G_{f}$ is the fracture energy, and $L_{c}$ represents characteristic element length (for a typical hexahedral mesh, it represents the shortest distance between diagonally opposite nodes of a smallest element). The elastic and strength properties for CFRP composite laminates were taken from [7] and [11]. The mechanical and shock-related properties for Al-HC core were obtained from the manufacturer's catalogue, and the Abaqus technical brief [10], respectively, owing to identical material system. These are listed in Table 1. 
Table 1. Material properties for Al 6061-T6 core.

\begin{tabular}{ll}
\hline \multicolumn{2}{l}{ For Mie-Grüneisen EOS model } \\
\hline Reference density $\left(\mathrm{kg} / \mathrm{m}^{3}\right)$ & 2700 \\
Grüneisen coefficient & 1.97 \\
Wave speed $(\mathrm{m} / \mathrm{s})$ & 5240 \\
Parameter, s & 1.40 \\
Reference temperature $(\mathrm{K})$ & 293.2 \\
Specific heat $(\mathrm{J} / \mathrm{kg} \mathrm{K})$ & 885 \\
\hline For Johnson Cook plasticity and failure model \\
\hline $\mathrm{A}$ & 324.1 \\
$\mathrm{~B}$ & 113.8 \\
$\mathrm{n}$ & 0.42 \\
$\theta_{\text {melt }}(K)$ & 925 \\
$\theta_{\text {transition }}(K)$ & 293.2 \\
$\mathrm{~m}$ & 1.34 \\
$\mathrm{c}$ & 0.002 \\
$\dot{\epsilon}_{0}(1 / \mathrm{s})$ & 1 \\
\hline
\end{tabular}

\subsubsection{Element deletion}

An element-deletion approach [11] used to remove the failed elements from the mesh was based on the magnitude of damage variables, $d_{i}=f_{t}, f_{c}, m_{t}, m_{c}$ as calculated using Eq. (7) applied to discrete damage modes in the composite laminate. The element was removed when the magnitude of any of the damage variables associated with the fibre failure reached $d_{\max }(=1)$, at an integration point of the element. When this condition was satisfied, the element was removed from the mesh, and it offered no subsequent resistance to deformation.

\subsection{Response of Al honeycomb}

A hydrodynamic response of $\mathrm{Al}$ honeycomb was accounted for using the Mie-Gruneisen equation of state, while its deviatoric response was modelled using the linear elastic and Johnson-Cook plasticity model available in ABAQUS/Explicit. An identical element deletion strategy was used to remove excessively deformed elements from the simulation as discussed in Sect. 2.1.3.

\section{Results and discussion}

Delamination patterns at the entry and exit sides of CFRP face sheets were studied using the developed FE model. The extent of delamination between adjacent plies after the projectile perforated the CFRP/Al-HC structure is shown in Figs. 3 and 4. It should be noted that though the FE model was quarter-symmetric, full delamination planes were obtained during the post-processing offering better observation of these results. The ply-level damage was also studied: here fibre damage/spallation was the dominant mode of damage. The fibre damage on the front and back faces of entry and exit side face sheets is shown in Figs. 5a and $b$. Shear-driven crushing of Al core directly below the point of impact was also evident from simulations. This

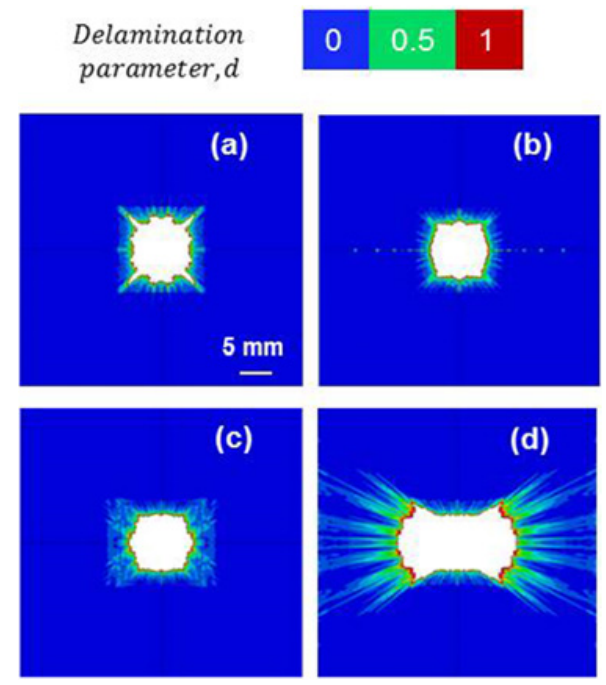

Figure 3. Entry-side delamination of CFRP face sheet between $0^{\circ}$ and $45^{\circ}(\mathrm{a}),-45^{\circ}$ and $45^{\circ}(\mathrm{b}), 45^{\circ}$ and $0^{\circ}(\mathrm{c}), 45^{\circ}$ and $-45^{\circ}$ (d) plies. $v_{i}=1 \mathrm{~km} / \mathrm{s}$, projectile radius $3 \mathrm{~mm}$.

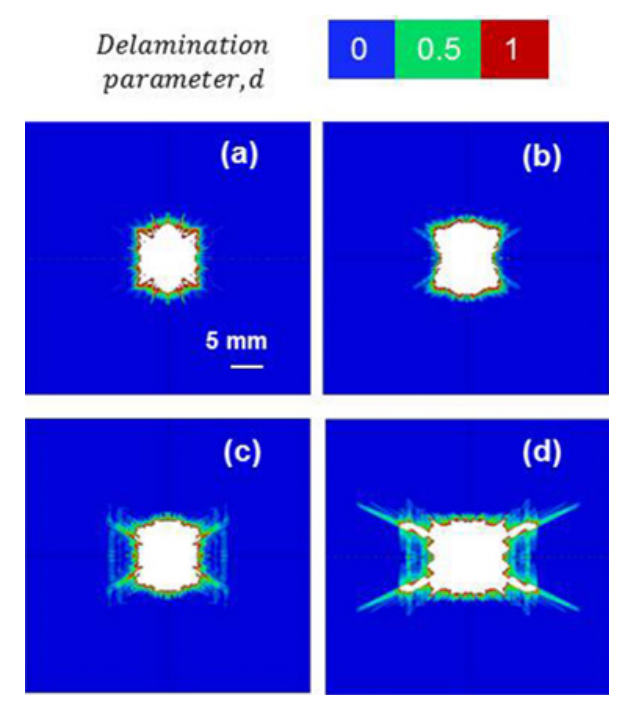

Figure 4. Exit-side delamination of CFRP face sheet between $0^{\circ}$ and $45^{\circ}(\mathrm{a}),-45^{\circ}$ and $45^{\circ}(\mathrm{b}), 45^{\circ}$ and $0^{\circ}(\mathrm{c}), 45^{\circ}$ and $-45^{\circ}(\mathrm{d})$ plies. $v_{i}=1 \mathrm{~km} / \mathrm{s}$, projectile radius $3 \mathrm{~mm}$.

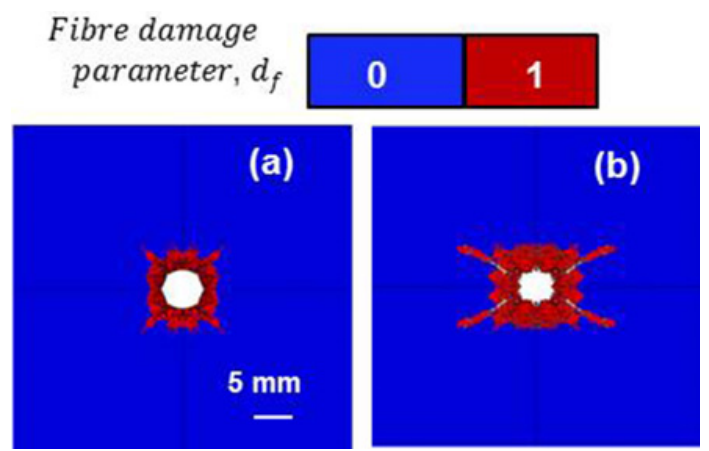

Figure 5. Fibre damage on surface of CFRP sheets at entry(a) and exit(b) sides. $v_{i}=1 \mathrm{~km} / \mathrm{s}$, projectile radius $3 \mathrm{~mm}$. 

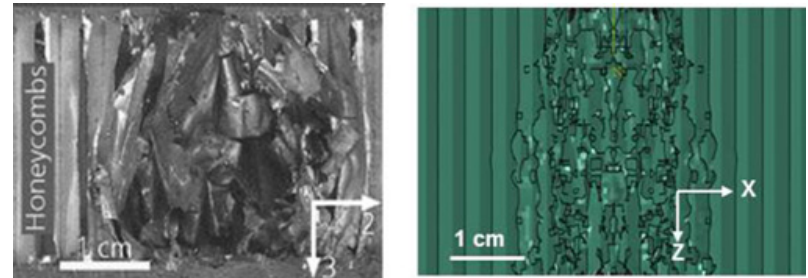

Figure 6. Damage of Al-HC core:comparison of experiment and FE analysis. $v_{i}=1 \mathrm{~km} / \mathrm{s}$, projectile radius $3 \mathrm{~mm}$.

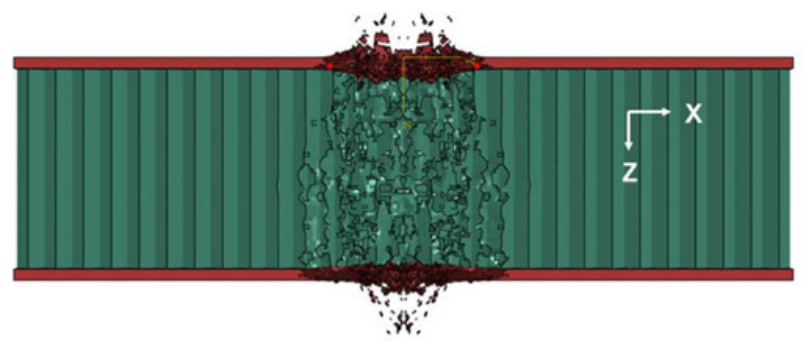

Figure 7. Cut view of final state of studied composite laminate showing surface peel up of CFRP face sheets. $v_{i}=1 \mathrm{~km} / \mathrm{s}$, projectile radius $3 \mathrm{~mm}$.

was compared with the experimental results of [7] and shown in Fig. 6 ( $\mathrm{X}-\mathrm{Z}$ plane of simulation corresponds to 2-3 plane of experimental results). The cut-view showing final configuration of CFRP/Al-HC composites structure is presented in Fig. 7.

\section{Conclusions}

In this work, the hypervelocity $\left(v_{i}=1 \mathrm{~km} / \mathrm{s}\right.$, projectile radius $3 \mathrm{~mm}$ ) impact response of the CFRP/Al-HC composite structure was studied using a developed $3 \mathrm{D}$ finite-element model. Damage to CFRP face sheets was observed in terms of dynamic spall and surface peeling, while Al-HC core failed due to core cracking driven by shear. The simulation results offer a reasonable agreement with experimental observations.

\section{References}

[1] Riedel, W., Harwick, W., White, D.M. \& Clegg, R.A. (2003) ADAMMO - advanced material damage models for numerical simulation codes. ESA CR(P) 4397, EMI report I 75/03, Freiburg.

[2] Riedel, W., Nahme, H., White, D.M. \& Clegg R.A. (2006) Hypervelocity impact damage prediction in composites: part II - experimental investigations and simulations. Int. J. Impact Engng 33, 670-80.

[3] Hiermaier. S., Riedel, W., Hayhurst, C., Clegg, R.A. \& Wentzel, C. (1999) AMMHIS - advanced material models for hypervelocity impact simulations. Final report, EMI report E 43/98, ESA CR(P) 4305, Freiburg.

[4] White, D.M., Wicklein, M., Clegg, R.A. \& Nahme, H. (2008) Multi-layer insulation material models suitable for hypervelocity impact simulations. Int. J. Impact Engng 35(12), 1853-1860.

[5] White, D.M., Taylor, E.A. \& Clegg, R.A. (2003) Numerical simulation and experimental characterization of direct hypervelocity impact on a spacecraft hybrid carbon fibre/Kevlar composite structure. Int. J. Impact Eng 29(1-6), 779-90.

[6] ABAQUS 6.10. Theory manual (2010) Dassault System.

[7] Wicklein, M., Ryan, S., White, D.M. \& Clegg, R.A. (2008) Hypervelocity impact on CFRP: Testing, material modelling, and numerical simulation. Int. J. Impact Engng 35: 1861-1869.

[8] Hashin, Z. (1980) Failure criteria for unidirectional fibre composites. J. Appl. Mech. 47: 321-329.

[9] Puck, A. \& Schürmann, H. (1998) Failure analysis of FRP laminates by means of physically based phenomenological models. Comp. Sci. Technol. 58(7): 1045-1067.

[10] Simulia Abaqus technical brief (2012) Simulation of the ballistic performance of aluminium plates with Abaqus/Explicit, USA.

[11] Phadnis, V.A., Makhdum, F., Roy, A. \& Silberschmidt, V.V. (2013) Drilling in carbon/epoxy composites: Experimental investigations and finite element implementation. Comp. Pt A 47: 41-51. 